\title{
Water Permeability Increase in Ultrafiltration Cellulose Acetate Membrane Containing Silver Nanoparticles
}

\author{
Bruna da Silva Pereira ${ }^{a}$, Livia Oliveira Ruiz Moreti ${ }^{b}$, Marcela Fernandes Silva ${ }^{b}$, Rosangela \\ Bergamasco $^{b}$, Angélica de Fátima Bortolato Piccioli ${ }^{a}$, Edvaldo Egea Garcia ${ }^{a}$, Wilian Vidoto \\ da Costa ${ }^{a}$, Edgardo Alfonso Gómez Pineda ${ }^{a}$, Daniela Martins Fernandes de Oliveira ${ }^{a}$, \\ Ana Adelina Winkler Hechenleitner ${ }^{a}$ * \\ ${ }^{a}$ Departamento de Química, Universidade Estadual de Maringa - UEM, Maringá, PR, Brasil \\ ${ }^{b}$ Departamento de Engenharia Química, Universidade Estadual de Maringa - UEM, \\ Maringá, PR, Brasil
}

Received: December 13, 2016; Revised: December 20, 2017; Accepted: December 23, 2017

\begin{abstract}
Inorganic nanoparticles incorporation in polymer membranes for filtration processes has attracted the attention of researchers in order to improve in some ways their performance. In this work, AgNPs were synthesized "in situ" in cellulose acetate (CA) membrane by chemical reduction of a silver salt. The membranes were prepared by the nonsolvent induced phase inversion method. Asymmetric membranes without (CA-M) and with adsorbed AgNPs (CA-Ag-M) were obtained. Ultrafiltration properties were confirmed for both membranes ( $16 \mathrm{~nm}$ of average pore diameter) and average crystallite size of $21 \mathrm{~nm}$ for the adsorbed AgNPs was computed. Contact angle measured with Milli-Q water and dry CA-Ag-M is near zero while for the CA-M is around $80^{\circ}$. The CA-M showed water permeability of $214 \mathrm{Lh}^{-1} \mathrm{~m}^{-2}$, while the CA-Ag-M permeability was $1651 \mathrm{Lh}^{-1} \mathrm{~m}^{-2}$.
\end{abstract}

Keywords: silver nanoparticles, cellulose acetate membrane, permeability, ultrafiltration, contact angle.

\section{Introduction}

Ultrafiltration (UF) process has been widely used in efficient separation technology ${ }^{1-3}$. UF membranes, with superficial pores ranging from 1 to $100 \mathrm{~nm}$, retain macromolecules with molar massin the range of 5 to $500 \mathrm{KDa}^{4}$. One of the polymers widely used to obtain these membranes is cellulose acetate (CA) because it is a good film forming material, of low cost, biocompatible, among others ${ }^{5}$.

Recently, several studies have been carried out with the aim of improving the performance of UF membranes ${ }^{6-9}$. Enhancing the hydrophilicity of the membrane surface is an effective method for improving its filtration properties ${ }^{4}$. Studies have shown that the adsorption of silver nanoparticles (AgNPs) onto membranes makes its surface more hydrophilic, allowing an increase in the flux that permeates the membrane, and consequently, its permeability ${ }^{10,11}$.

$\mathrm{Li}$ and co-workers prepared a polyvinylidenefluoride (PVDF) membrane with $\mathrm{Ag} / \mathrm{TiO}_{2}$ nanoparticles, and using contact angle measurements, they observed that silver increased the membrane hydrophilicity ${ }^{11}$. Dong, et al. incorporated AgNP sin situ to a commercial polyamide reverse osmosis membrane and water contact angle measurement also indicated increased surface hydrophilicity after AgNPs incorporation. Compared with commercial polyamide membrane, the water

*e-mail: anitawh@uem.br flux that permeates the membrane with silver increased from approximately 45 to $55 \mathrm{Lh}^{-1} \mathrm{~m}^{-2}$ and pressure of $1.55 \mathrm{MPa}^{10}$.

In the present work, cellulose acetate membrane with adsorbed AgNPs (CA-Ag-M) was obtained by the method of phase inversion and chemical reduction of a silver salt, respectively. In this way, the AgNPs are formed and adsorbed at the external surface of the membrane causing a change of the chemical composition of this surface layer providing also possible surface properties alterations. The obtained membranes were characterized by scanning electron microscopy (SEM), $\mathrm{X}$-ray diffraction (XRD) and measurements of thickness, porosity, specific surface area, pore diameter and volume, water contact angle and permeability. Silver release from this membrane in anone hour of water filtration experiment was also measured.

\section{Experimental}

\subsection{Materials}

Cellulose acetate $\left(\mathrm{Mw} 50000 \mathrm{~g} \mathrm{~mol}^{-1}, 39.7 \mathrm{~g} \mathrm{~mol}^{-1}\right.$ $\%$ acetyl) and polyvinylpyrrolidone (Mw 1300000) were purchased from Sigma-Aldrich. Acetone, glacialacetic acid and silver nitrate were purchased from Synth. Hydroxylamine hydrochloride was purchased from Vetec. Ammonium hydroxide was purchased from Nuclear.These materials 
were used as received without further purification.Other materials as chrome nickel wire (thickness of $0.4 \mathrm{~mm}$ ), glass plate $(20 \times 20 \mathrm{~cm})$, distilled and Milli-Q water were used.

\subsection{CA-M preparation}

Initially a solution containing $10 \%(\mathrm{w} / \mathrm{w})$ cellulose acetate, $17 \%(\mathrm{w} / \mathrm{w})$ acetic acid, $23 \%(\mathrm{w} / \mathrm{w})$ water and $50 \%$ (w/w) acetone was prepared. Posteriorly, near $20 \mathrm{~mL}$ of this solution was distributed with a glass bar on a glass plate containing two stretched nickel-chromium wires ( 0.4 $\mathrm{mm}$ thick) to allow membrane thickness control. After five minutes of solvent evaporation, the glass plate was introduced into a recipient containing distilled water at temperature of $10{ }^{\circ} \mathrm{C}$ for polymer coagulation. The obtained membrane (CA-M) was kept in a recipient (with distilled water) at temperature of approximately $10^{\circ} \mathrm{C}$, or lyophilized for future characterizations. After membrane lyophilization, the surface in contact with the glass was identified as opaque $(\mathrm{O})$, and the surface in contact with air as brilliant (B).

\section{3. "In situ" synthesis of AgNPs onto CA-M}

AgNPs were synthesized on the CA-M surface using a methodology employed previously by our group ${ }^{12}$. Three aqueous solutions were prepared separately: $0.06 \mathrm{molL}^{-1}$ silver nitrate- $\mathrm{AgNO}_{3}$ and $0.2 \% \mathrm{~m} / \mathrm{v}$ polyvinylpyrrolidonePVP (solution I), 0.2\% w/v PVP and ammonium hydroxide$\mathrm{NH}_{4} \mathrm{OH}(\mathrm{pH} 12.5$ ) (solution II) and $0.2 \% \mathrm{w} / \mathrm{v}$ PVP and 0.002 molL ${ }^{-1}$ hydroxylamine reducing agent $\mathrm{NH}_{2} \mathrm{OH}$ (solution III). $1 \mathrm{~g}$ of the wet membrane was placed in contact with $0.1 \mathrm{~L}$ of solution I for 24 hours at room temperature. Subsequently this material was removed and immersed in $0.1 \mathrm{~L}$ of solution II and maintained at room temperature with stirring. Then, $0.05 \mathrm{~L}$ of solution III was added dropwise until the total reduction of silver, which was observed visually. The obtained membranes (CA-Ag-M) were washed with distilled water and kept in a container (with distilled water) at a temperature of approximately $10^{\circ} \mathrm{C}$, or lyophilized for further characterizations.

\subsection{Characterization of the $C A-M$ and $C A-A g-M$}

The X-ray diffractograms (XRD) of the opaque surface of CA-M and CA-Ag-M were obtained using a SHIMADZU D6000 spectrometer, and a tube with $\mathrm{Cu} \mathrm{K \alpha}$ radiation of $1.54 \AA$. The experimental conditions used were: $2 \theta=10-80^{\circ}$ and $2^{\circ} \mathrm{min}^{-1}$. The average diameter of the silver crystallites (d) was estimated by the Scherrer equation ${ }^{13}$ using the silver characteristic peak at $2 \Theta=38.1^{\circ}$, and Equation $\mathrm{A}$.

$$
d=\frac{0.9 \lambda}{B \cos \theta_{B}}
$$

In this equation, $\lambda$ is the wavelength of the $\mathrm{X}$-rays, $\mathrm{B}$ is the half-height width of the sample peak, and $\Theta_{B}$ is the corresponding Bragg angle.
Scanning electron microscopy (SEM) images of opaque, brilliant, and cross-section surface of the membranes without and with silver, were obtained using a SHIMADZU SSX-550 Supercan microscope. SEM images of the membrane cross section were obtained from samples fractured immediately after immersion in liquid nitrogen. The samples were prepared according to a standard procedure, fixed with conductive paste and coated with a thin layer of gold.

For membranes porosity evaluation, small pieces (approximately $4 \mathrm{~cm}^{2}$ ) absorbed with distilled water were cut out. These were dried on a paper towel and weighed. They were then placed in a recipient, and it was kept in an incubator at $80^{\circ} \mathrm{C}$ for $24 \mathrm{~h}$ to dry completely. Subsequently, the membrane pieces were weighed again. Membranes porosity was calculated using Equation $\mathrm{B}^{14}$ :

$$
P(\%)=\left(\frac{Q_{0}-Q_{1}}{A \cdot h}\right) \cdot 100
$$

In equation $\mathrm{B}, \mathrm{P}$ is the porosity $(\%), \mathrm{Q}_{0}$ is the mass of the wet membrane $(\mathrm{g}), \mathrm{Q}_{1}$ is the mass of the dry membrane $(\mathrm{g})$, A is the wet membrane area $\left(\mathrm{cm}^{2}\right)$ and $\mathrm{h}$ is the wet membrane thickness $(\mathrm{cm})$. Measurements were performed in triplicate.

The measurements of the specific surface area, pore diameter and volume of the membranes were realized by $\mathrm{N}_{2}$ adsorption usinga QUANTA CHROME - Nova 1000 seriesinstrument, using the BET (Brunauer-Emmet-Teller) and BJH (Barret-Joyner-Halenda) methods. Approximately $9.8 \mathrm{mg}$ of each sample was treated with $\mathrm{N}_{2}$ flux.

Contact angle measurements of Milli-Q water onto lyophilized CA membranes were performed using Tantec Contact Angle Meter - Cam-Micro model.

\subsection{Performance of the $C A-M$ and $C A-A g-M$}

A dead-end ultrafiltration system was used in this work. The system consists of a selective membrane module with area of $2.58 \mathrm{~cm}^{2}$, $4 \mathrm{~L}$ of water capacity coupled with a $\mathrm{N}_{2}$ cylinder.

All membraneswere pressurized with Milli-Q water at 1.5 bar for 15 minutes before flux measurement.At pressures of $1.0 ; 1.5 ; 2.0 ; 2.5$ and 3.0 bar the Milli-Q water flux that permeates the membrane was measured. In these measurements, the time required to collect $2 \mathrm{~mL}$ of water was verified. The analysis was performed in duplicate. The water flux was calculated according to Equation C.

$$
J=\left(\frac{\Delta V}{A . \triangle t}\right)
$$

In this equation, $\mathrm{J}$ is the water flux $\left(\mathrm{Lh}^{-1} \mathrm{~m}^{-2}\right), \Delta \mathrm{V}$ is the permeate volume (L), $\mathrm{A}$ is the membrane area $\left(\mathrm{m}^{2}\right)$ and $\Delta \mathrm{t}$ is the permeation time $(\mathrm{h})$.

Silver liberation from CA-Ag-M in the permeated water was evaluated using Milli-Q water, for $1 \mathrm{~h}$ of filtration period at pressure of 2.5 bar. Aliquots were removed every 15 minutes and the result was obtained by averaging the 4 measurements. Permeate absorbance measurements were performed on a 
SpectrAAS-240FS atomic absorption spectrometer equipped with a deuterium lamp-based background signal correction system coupled to a graphite furnace with longitudinal heating (GTA-120) and an automatic sampler (PSD-120), all from Varian ${ }^{\circledR}$. The measurements were carried out on partition tubes type pyrolytic graphite tubes (Part Number 63-100012-

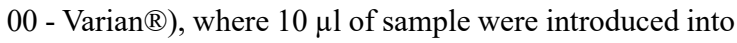
the tube, accompanied by $5 \mu \mathrm{L}$ of a chemical modifier. A multielement hollow cathode ultra-lamp (Ag, $\mathrm{Cd}, \mathrm{Pb}$ and $\mathrm{Zn}$ ) was used as radiation source.

\section{Results and Discussion}

\subsection{Characterization of the $C A-M$ and $C A-A g-M$}

Silver nanoparticles were synthesized in situ onto the CA-M surface. A silver salt solution was used for the silver ions to diffuse until CA-M surface. Polyvinylpyrrolidone was used to control the silver growing particle diameter as well as their stability. Sodium hydroxide was used to accelerate the synthesis reaction and to allow smaller particle diameters. Studies have shown that $\mathrm{pH}$ influences the diameter of the particles, higher $\mathrm{pHs}$ infer in smaller particle diameter ${ }^{15}$.

The XRD graphs of the prepared membranes opaque surface are shown in Figure 1. A typical curve of amorphous CA was observed in the region of $2 \theta=10$ to $30^{\circ}$. The CA$\mathrm{Ag}-\mathrm{M}$ diffractogram indicate the formation of silver in the region of $2 \theta=38.1 ; 44.3$ and $64.4^{\circ}$ which can be attributed to the crystallographic planes (111), (200) and (220), respectively. These peaks are characteristic of silver with face-centered cubic structure (PCPDF\# 893722). According to the Scherrer equation, the estimated average diameter of the silver crystallites adsorbed on the membrane (using (111) reflection plane) was $21 \mathrm{~nm}$.

Scanning electron microscopy (SEM) images of the opaque surface, brilliant surface and cross section of the CA-M and CA-Ag-M were obtained (Figure 2). It can be noted that the filtering layer or brilliant surface of the membranes (Fig. 2 A and D) is slightly rough and homogeneous without visible pores, where as at the supporting layer or opaque surface (Fig. 2B and E) pores with diameters around $20 \mathrm{~nm}$ can be seen. SEM images of the membranes cross section (Fig. $2 \mathrm{C}$ and $\mathrm{F}$ ) show the fine filtering layer as well as the thick supporting layer in both asymmetric membranes. It is also possible to observe distributed particles at both surfaces of the CA-Ag-M images (D and E) which correspond to AgNPs.

The thickness of the CA membranes were measuredand the results are shown in Table 1. As expected, silver adsorption on the membrane did not alter its thickness, while lyophilization reduces its thickness in approximately $50 \%$.

Specific surface area as well as parameters such as pore diameter and volume can be estimated from the $\mathrm{N}_{2}$ adsorption isotherm, using the BET and BJH methods (see Table 2). It is possible to observe a small decrease in the

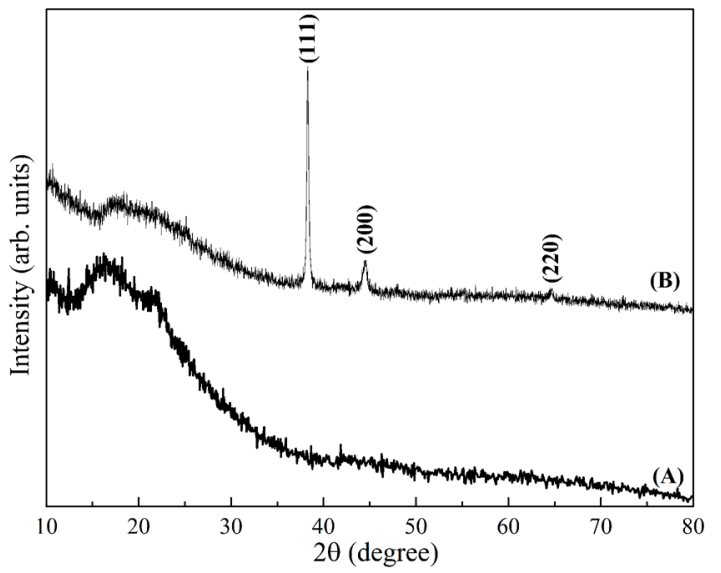

Figure 1. X-ray diffractograms of the opaque surface of the prepared (A) CA-M and (B) CA-Ag-M.
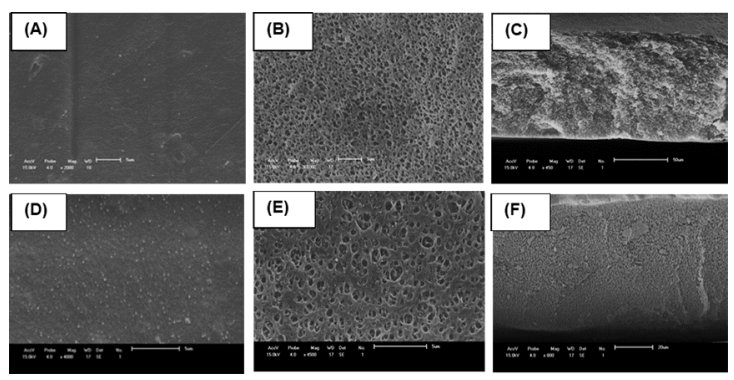

Figure 2. SEM images from brilliant surface (A), opaque surface (B) and cross section (C) of the CA-M. SEM images from brilliant surface (D), opaque surface (E) and cross section (F) of the CA-Ag-M.

Table 1. Thickness of the studied membranes.

\begin{tabular}{lc}
\hline Sample & Thickness $(\boldsymbol{\mu m})$ \\
\hline Wet CA-M & $146.5 \pm 0.8$ \\
Dry CA-M & $72.0 \pm 1$ \\
Wet CA-Ag-M & $146.6 \pm 0.4$ \\
Dry CA-Ag-M & $71.9 \pm 0.6$ \\
\hline
\end{tabular}

specific surface area, and in the pore volume and diameter of CA-Ag-M compared to CA-M, probably due to the volume occupied by the AgNPs.

The membranes porosity was also measured and CA-Ag-M as well as CA-M presented similar results, nearly $13.6 \pm 0.4$. Porosity did not vary because AgNPs adsorb preferentially at the outer surfaces and not at the internal pores.

Furthermore, measurements of the contact angle of water onto dry CA-M and CA-Ag-M were measured. The contact angle of CA-M was $80 \pm 2^{\circ}$, however for the CA-Ag-M it was not possible to measure because the drop spread fully, showing that in situ adsorbed AgNPs provided a very hydrophilic surface. This property is very important for increasing the membrane permeability.

\subsection{Performance of the $C A-M$ and $C A-A g-M$}

Results of permeability tests performed with the obtained membranes can be seen in Figure 3. This parameter increases 
Table 2. Results obtained from the $\mathrm{N}_{2}$ adsorption isotherm of the membranes.

\begin{tabular}{lccc}
\hline Sample & $\begin{array}{c}\text { Specific } \\
\text { surface area } \\
\mathbf{m}^{2} / \mathbf{g}\end{array}$ & $\begin{array}{c}\text { Volume } \\
\mathbf{c m}^{3} / \boldsymbol{g}\end{array}$ & $\begin{array}{c}\text { Pore } \\
\text { diameter } \\
\mathbf{n m}\end{array}$ \\
\hline CA-M & 90.29 & 29.74 & 16 \\
CA-Ag-M & 87.34 & 25.67 & 10 \\
\hline
\end{tabular}

from 214 to $1651 \mathrm{Lh}^{-1} \mathrm{~m}^{-2}$ after adsorption of AgNPs on the membrane. Since there is no change in average pore diameter of the membranes, this phenomenon can be explained based on the hydrophilic surface that the AgNPs cause on the membrane, allowing the water flux to be more permeable in CA-Ag-M when compared to the CA-M.
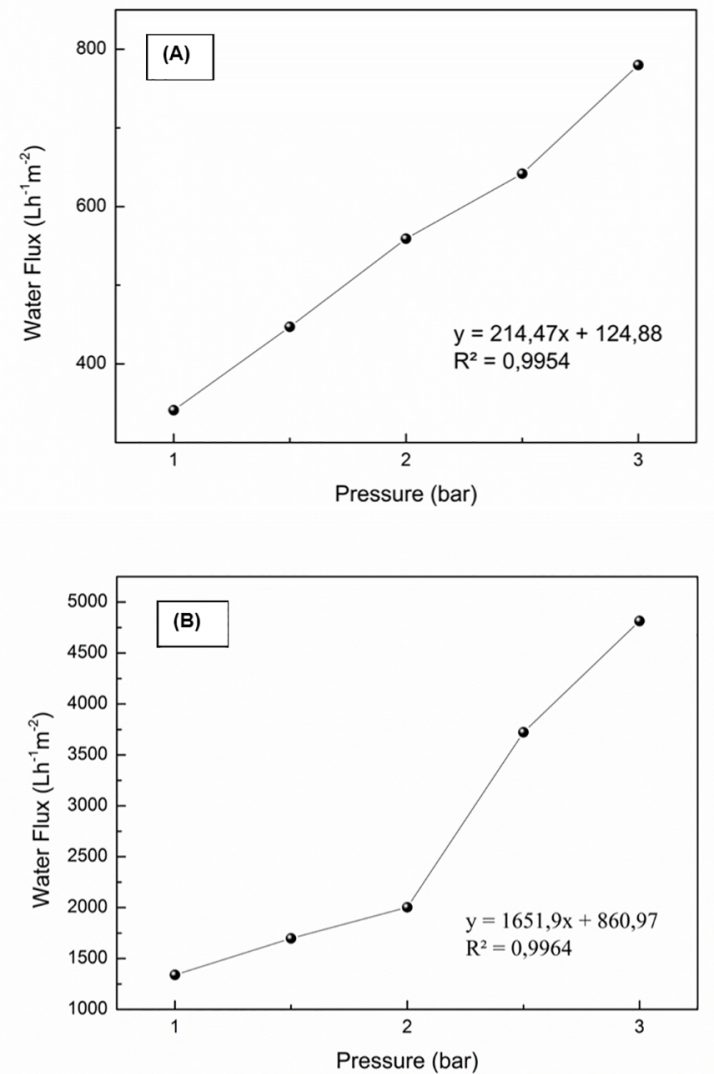

Figure 3. Water permeability using Milli-Q water of the (A) CA-M and (B) CA-Ag-M.

A study was carried out regarding the release of silver incorporated into the membranes during the water filtration process. Silver content in permeated water was determined by means of atomic absorption with graphite furnace. Initially, a calibration curve was constructed with silver concentration in the range from 0.25 to $20.00 \mu \mathrm{g} \mathrm{L}^{-1}$ (see Figure 4). In the water collected after a period of one hour of filtration through the CA-Ag-M, a silver concentration of $0.12 \mu \mathrm{g} \mathrm{L}^{-1}$ was detected. This value is much lower than the maximum limit of silver contaminants in drinking water $\left(100 \mu \mathrm{g} \mathrm{L}^{-1}\right)$ proposed by the regulation of the environmental protection agency and the guidelines of the World Health Organization.

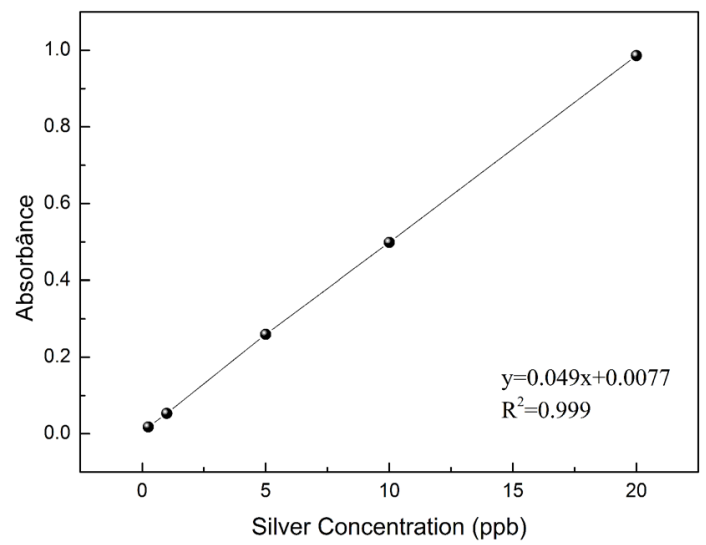

Figure 4. Calibration curve for concentration of silver solutions determination from atomic absorption measurements.

\section{Conclusion}

Asymmetric cellulose acetate membranes (CA-M and CA-Ag-M) were prepared and characterized. The membranes were obtained by the phase inversion method and AgNPs were deposited by a chemical reduction method of a silver salt. Based on the average pore diameter $(16 \mathrm{~nm})$ and water permeability at 1-3 bar of applied pressure (214 to $1651 \mathrm{Lh}^{-}$ ${ }^{1} \mathrm{~m}^{-2}$ ) data, CA-M and CA-Ag-M are classified as ultrafiltration membranes. AgNPs with a mean crystallite diameter of $21 \mathrm{~nm}$ distributed homogeneously were observed on the surface of CA-Ag-M. AgNPs modify the membrane surface making it hydrophilic, increasing eight times the permeation flux when compared with the CA-M. The release of silver from the CA-Ag-M in the permeate in one hour water filtration was very small, the silver concentration determined by atomic absorption was lower than the maximum limit of silver contaminants in drinking water $\left(100 \mu \mathrm{g} \mathrm{L}^{-1}\right)$ established by the regulation of the environmental protection agency and the guidelines of the World Health Organization.

\section{Acknowledgment}

The authors gratefully acknowledge the financial support of CAPES (Coordenação de Aperfeiçoamento de Pessoal de Nível Superior).

\section{References}

1. Kasemset S, Wang L, He ZH, Miller DJ, Kirschner A, Freeman $\mathrm{BD}$, et al. Influence of polydopamine deposition conditions on hydraulic permeability, sieving coefficients, pore size and pore size distribution for a polysulfone ultrafiltration membrane. Journal of Membrane Science. 2017;522:100-115. DOI: 10.1016/j.memsci.2016.07.016 
2. Rabiee H, Shahabadi SMS, Mokhtare A, Rabiei H, Alvandifar $\mathrm{N}$. Enhancement in permeation and antifouling properties of PVC ultrafiltration membranes with addition of hydrophilic surfactant additives: Tween-20 and Tween-80. Journal of Environmental Chemical Engineering. 2016;4(4 Pt A):40504061. DOI: 10.1016/j.jece.2016.09.015

3. Nayak V, Geetha Balakrishna R, Padaki M, Soontarapa K. Zwitterionic ultrafiltration membranes for As (V) rejection. Chemical Engineering Journal. 2017;308:347-358. DOI: 10.1016/j.cej.2016.09.096

4. Huang L, Zhao S, Wang Z, Wu J, Wang J, Wang S. In situ immobilization of silver nanoparticles for improving permeability, antifouling and anti-bacterial properties of ultrafiltration membrane. Journal of Membrane Science. 2016;499:269-281. DOI: 10.1016/j.memsci.2015.10.055

5. Mai-Gisondi G, Turunen O, Pastinen O, Pahimanolis N, Master ER. Enhancement of acetyl xylan esterase activity on cellulose acetate through fusion to a family 3 cellulose binding module. Enzyme and Microbial Technology. 2015;79-80:27-33. DOI: 10.1016/j.enzmictec.2015.07.001

6. Luo L, Chung TS, Weber M, Staudt C, Maletzko C. Molecular interaction between acidic SPPSU and basic HPEI polymers and its effects on membrane formation for ultrafiltration. Journal of Membrane Science. 2017;524:33-42. DOI: 10.1016/j. memsci.2016.11.016

7. Torres JJ, Rodriguez NE, Arana JT, Ochoa NA, Marchese J, Pagliero C. Ultrafiltration polymeric membranes for the purification of biodiesel from ethanol. Journal of Cleaner Production. 2017;141:641-647. DOI: 10.1016/j.jclepro.2016.09.130

8. Bouazizi A, Breida M, Karim A, Achiou B, Ouammou M, Calvo JI, et al. Development of a new $\mathrm{TiO}_{2}$ ultrafiltration membrane on flat ceramic support made from natural bentonite and micronized phosphate and applied for dye removal. Ceramics International. 2017;43(1 Pt B):1479-1487. DOI: 10.1016/j. ceramint.2016.10.118
9. Lin CE, Wang J, Zhou MY, Zhu BK, Zhu LP, Gao CJ. Poly(mphenyleneisophthalamide) (PMIA): A potential polymer for breaking through the selectivity-permeability trade-off for ultrafiltration membranes. Journal of Membrane Science. 2016;518:72-78. DOI: 10.1016/j.memsci.2016.06.042

10. Dong C, Wang Z, Wu J, Wang Y, Wang J, Wang S. A green strategy to immobilize silver nanoparticles onto reverse osmosis membrane for enhanced anti-biofouling property. Desalination. 2017;401:32-41. DOI: 10.1016/j.desal.2016.06.034

11. Li JH, Yan BF, Shao XS, Wang SS, Tian HY, Zhang QQ. Influence of $\mathrm{Ag} / \mathrm{TiO}_{2}$ nanoparticle on the surface hydrophilicity and visible-light response activity of polyvinylidene fluoride membrane. Applied Surface Science. 2015;324:82-89. DOI: 10.1016/j.apsusc.2014.10.080

12. Pereira BS, Silva MF, Bittencourt PRS, Oliveira DMF, Pineda EAG, Hechenleitner AAW. Cellophane and filter paper as cellulosic support for silver nanoparticles and its thermal decomposition catalysis. Carbohydrate Polymers. 2015;133:277-283. DOI: 10.1016/j.carbpol.2015.06.108

13. Langford JI, Wilson AJC. Scherrer after sixty years: A survey and some new results in the determination of crystallite size. Journal of Applied Crystallography. 1978;11:102-113. DOI: $10.1107 / \mathrm{S} 0021889878012844$

14. Zheng QZ, Wang P, Yang YN, Cuid DJ. The relationship between porosity and kinetics parameter of membrane formation in PSF ultrafiltration membrane. Journal of Membrane Science. 2006;286(1-2):7-11. DOI: 10.1016/j.memsci.2006.09.033

15. Ajitha B, Reddy YAK, Reddy PS. Enhanced antimicrobial activity of silver nanoparticles with controlled particle size by pH variation. Powder Technology. 2015;269:110-117. DOI: 10.1016/j.powtec.2014.08.049 